\title{
The Impact of Sequence of Chemotherapy and EGFR-TKI Treatment on Different EGFR Mutation Lung Adenocarcinoma
}

\author{
Fu-Tsai Chung, ${ }^{1,2,3,4}$ Ming-Yun Ho, ${ }^{4}$ Yueh-Fu Fang, ${ }^{2,4}$ Meng-Heng Hshieh, \\ Tsai-Yu Wang, ${ }^{2,4}$ Chih-Hsi Kuo, ${ }^{2,4}$ Hao-Cheng Chen, ${ }^{3}$ Chun-Hwa Wang, ${ }^{2,4}$ Shu-Min Lin, ${ }^{2,4}$ \\ Chih-Teng Yu, ${ }^{2,4}$ Horng-Chyuan Lin, ${ }^{2,4}$ and Han-Pin Kuo ${ }^{2,4}$
}

\author{
${ }^{1}$ Department of Thoracic Medicine, Saint Paul's Hospital, Taoyuan 330, Taiwan \\ ${ }^{2}$ Department of Thoracic Medicine, Chang Gung Memorial Hospital at Linkou, Chang Gung University, College of Medicine, \\ Taipei 10507, Taiwan \\ ${ }^{3}$ Department of Internal Medicine, Saint Paul's Hospital, Taoyuan 330, Taiwan \\ ${ }^{4}$ Department of Internal Medicine, Chang Gung Memorial Hospital at Linkou, Chang Gung University, College of Medicine, \\ Taipei 10507, Taiwan
}

Correspondence should be addressed to Fu-Tsai Chung; vikingchung@yahoo.com.tw and Han-Pin Kuo; q8828@ms11.hinet.net

Received 16 April 2015; Accepted 22 July 2015

Academic Editor: Sherven Sharma

Copyright (C) 2015 Fu-Tsai Chung et al. This is an open access article distributed under the Creative Commons Attribution License, which permits unrestricted use, distribution, and reproduction in any medium, provided the original work is properly cited.

Objectives. Chemotherapy as first-/second-line treatment in different epidermal growth factor receptor (EGFR) mutation lung adenocarcinoma remains controversial. Methods. Consecutive patients were collected between 2009 and 2012. Patients were divided into two groups (1st-line chemotherapy: $n=56$ and 2nd-line chemotherapy: $n=55$ ). Their outcomes profiles were analyzed. Results. The overall survival (OS) of all patients (390 versus 662 days, $p<0.0001$ ), as well as both progression-free survival (PFS, 151 versus 252 days, $p=0.0001)$ and OS (308 versus 704 days, $p=0.0001)$ of patients with $L 858 R$ mutation $(n=63)$, who received 2 ndline chemotherapy, was significantly poor. By univariate and multivariate analysis, 2nd-line chemotherapy, and $L 858 R$ mutation were significantly related to poor PFS and OS. Conclusion. In advanced lung adenocarcinoma, L858R mutation and 2nd-line chemotherapy caused a poor outcome. It is a consideration to choice of 1st-line chemotherapy in these subjects. A prospective design is warranted to confirm this finding.

\section{Introduction}

The major forms of lung cancer are non-small-cell lung cancer (NSCLC) and small-cell lung cancer. NSCLC is divided into three subtypes: squamous-cell carcinoma, adenocarcinoma, and large-cell lung cancer. Most of lung cancer in nonsmoker group is adenocarcinoma [1]. It is often diagnosed at advanced stage and has poor prognosis. The systemic therapy is appropriated for advanced lung cancer. The choice of systemic therapy includes chemotherapy or the agents to specific molecular pathway. The option of treatment depends upon tumor histology, stage, molecular characteristics, performance status, and underlying diseases [1].

The mutant epidermal growth factor receptor (EGFR) is a specific molecular pathway in NSCLC [2]. The mutant
EGFR in adenocarcinoma lung cancer is approximately $10 \%$ in United States and 30 50\% in Asia [3]. The tyrosine kinase inhibitors (TKIs) such as gefitinib and erlotinib are target to EGFR. Those agents are oral active form and effective for advanced NSCLC $[4,5]$. They can preferentially affect malignant cells but less to affect normal cells.

In recent years, the clinical benefits of TKIs were discovered in EGFR mutation, nonsmokers, women, adenocarcinoma, and Asian ethnicity patients [6, 7]. The 1st-line TKIs in mutant EGFR NSCLC had better progression-free survival (PFS) than chemotherapy. However, there was no significant finding in overall survival (OS) $[7,8]$. It may relate to further systemic therapy after disease progression.

The patients with advanced NSCLC can receive 1st-line TKIs or chemotherapy followed by 2nd-line chemotherapy or 
TKIs as alternative treatment. The choice of 1st-line therapy between chemotherapy or TKIs is controversial. Despite the better PFS in TKIs therapy, the OS was still not significant in many trials $[8,9]$. There are less available data about the sequence of TKIs or chemotherapy in NSCLC. The different sequence of systemic therapy may have different efficiency.

In this study, the mutant EGFR advanced lung adenocarcinoma patients were collected from the cohort database. The efficiency between 1st chemotherapy/2nd TKIs and 1st TKIs/2nd chemotherapy group was compared. From the cohort database survey of mutant EGFR lung adenocarcinoma, we conducted this study to evaluate the response and survival in different sequence of chemotherapy/TKIs among all and different EGFR mutation subgroup patients.

\section{Methods}

2.1. Data Collection. The patients were included from Linkou Chang Gung Memorial Hospital and Saint Paul's Hospital at Taoyuan, Taiwan. The inclusion criteria were advanced lung adenocarcinoma with mutant EGFR patients, who received TKIs or platinum-based doublet chemotherapy as the 1stline treatment. The data was collected between 2009 and 2012. Patients received two kinds of therapies (EGFR-TKIs and chemotherapy) at different sequence were included. They were divided into two groups, 1st-line TKIs/2nd-line chemotherapy and 1st-line chemotherapy/2nd-line TKIs. The general data including age, smoking status, gender, performance status (PS), and EGFR mutation status before treatment were surveyed. The response after treatment was evaluated according to RECIST (Response Evaluation Criteria In Solid Tumors) criteria. The PFS and OS were investigated in two groups. The institutional review board of the institute approved the study.

2.2. Response. The tumor response to chemotherapy was assessed with computed tomography every two to three cycles, but tumor response was also evaluated at any time if signs of tumor progression were noted. Objective responses of tumor to chemotherapy were based on the Response Evaluation Criteria in Solid Tumors (RECIST) [10]. Partial response (PR) was defined as at least a 30\% decrease in the sum of diameters of target lesions, using the baseline sum diameters as a reference. Progressive disease (PD) was defined as at least a $20 \%$ increase in the sum of diameters of target lesions, using the smallest sum of the study as a reference (this includes the baseline sum if it is the smallest of the study). In addition to the relative increase of $20 \%$, the sum must also demonstrate an absolute increase of at least $5 \mathrm{~mm}$. The appearance of one or more new lesions was also considered progression. Stable disease (SD) was defined as neither sufficient shrinkage to qualify for PR nor a sufficient increase to qualify for $\mathrm{PD}$, with respect to the smallest sum of diameters while in the study.

2.3. Survival Evaluation. PFS and OS were estimated in two different sequences of TKIs/chemotherapy group. The OS from each arm of treatment was calculated from the start date of the treatment until death or until the last follow-up visit.
OS took all deaths into account. PFS included the time from the first cycle of chemotherapy or the date of TKIs treatment to documented progression until death from any cause or until the date of the last follow-up visit for patients who were still alive.

2.4. Determination of EGFR Mutations. The method to detect mutation was reported in our previous report [11]. Briefly, tissue sections from formalin fixed and paraffin embedded specimens were examined for EGFR mutations using the EGFR PCR Kit (QIAGEN Inc., Valencia, CA) according to the manufacturer's instruction. By combining two technologies, ARMS and Scorpions, this kit enabled the detection of the most prevalent somatic mutations in the EGFR gene, which were common in human cancers. Surgically resected tissue samples containing tumor cell proportion of at least $10 \%$ or biopsy samples of at least 30\% were determined as adequate. Tumor cell enrichment was achieved using Laser capture microdissection for those with $<60 \%$ tumor cell proportion while DNA was extracted by the QIAamp DNA FFPE Tissue Kit (QIAGEN Inc., Valencia, CA). QPCR was performed in a Rotor-Gene $\mathrm{Q}$ real-time PCR cycler (QIAGEN Inc., Valencia, CA) using the protocol provided by the EGFR PCR Kit. Samples with repeated negative control reactions were interpreted as inadequate or poor quality DNA.

2.5. Statistical Analyses. The baseline characteristics of patients, including age, sex, smoking history, and PS were analyzed by $t$-test or Chi-square test with a contingency table. OS and PFS were both analyzed in this study. Survival curves were estimated by the Kaplan-Meier method, whereas the log-rank test was used to compare the patient survival times per group. OS was calculated from the start date of treatment until patient death. PFS included the time from the start of treatment to documented progression until death from any cause. The Cox proportional model was fit to estimate the hazard ratio (HR) for the calculated risk factors. Univariate and multivariate analyses were performed to identify independent factors contributing to PFS and OS in all patients, and in those with EGFR (L858R) mutation to control for possible confounding factors. Univariate analyses were primarily used to select variables based on $p<0.05$. These variables were then entered into a multinominal logistic regression analysis to identify the net effects of each individual factor. The median PFS and median OS were used as dependent variables in analyzing contributing factors to PFS and OS, respectively. Odds ratios (OR) and their 95\% confidence intervals (CI) were computed with a logistic regression model to clarify the impact of several potentially independent prognostic factors. Statistical analysis was performed using SPSS software (version 10.0, SPSS, Chicago, IL, USA) and Prism 5 for Windows (version 5.01, Graphpad Software, Inc., San Diego, CA, USA), and statistical significance was considered at $p<0.05$.

\section{Results}

3.1. Patients. During the study period, 468 patients with advanced non-small-cell lung cancer were surveyed. We 
TABle 1: Demography.

\begin{tabular}{|c|c|c|c|}
\hline Characteristic & $\begin{array}{l}\text { 1st TKIs/2nd chemotherapy } \\
\qquad(n=55)\end{array}$ & $\begin{array}{l}\text { 1st chemotherapy/2nd TKIs } \\
\qquad(n=56)\end{array}$ & $p$ value \\
\hline Age, yr, median (IQR)* & $59(52-69)$ & $58(52-64)$ & 0.15 \\
\hline Female, $n(\%)$ & $30(54.5 \%)$ & $27(48.2 \%)$ & 0.5 \\
\hline Smoking, $n(\%)$ & $25(45.5 \%)$ & $17(30.4 \%)$ & 0.1 \\
\hline \multicolumn{4}{|c|}{ Performance status at beginning of 1st-line treatment } \\
\hline $0-1$ & $42(76.4 \%)$ & $47(83.9 \%)$ & 0.32 \\
\hline 2 & $9(16.4 \%)$ & $8(14.3 \%)$ & 0.76 \\
\hline$>2$ & $4(7.3 \%)$ & $1(1.8 \%)$ & 0.16 \\
\hline \multicolumn{4}{|l|}{ EGFR mutation status } \\
\hline $19 \mathrm{Del}$ & $21(38.1 \%)$ & $23(41.0 \%)$ & \\
\hline$L 858 R$ & $32(58.2 \%)$ & $31(55.4 \%)$ & 0.74 \\
\hline Others & $2(3.7 \%)$ & $2(3.6 \%)$ & \\
\hline \multicolumn{4}{|c|}{ Progression site from each 1st-line treatment } \\
\hline Primary lung & $17(30.9 \%)$ & $19(33.9 \%)$ & 0.73 \\
\hline Lung to lung metastasis & $10(18.2 \%)$ & $9(16.1 \%)$ & 0.77 \\
\hline Bone & $6(10.9 \%)$ & $9(16.1 \%)$ & 0.43 \\
\hline Pleural effusion & $6(10.9 \%)$ & $7(12.4 \%)$ & 0.79 \\
\hline CNS & $9(16.4 \%)$ & $5(8.9 \%)$ & 0.52 \\
\hline Liver & $3(5.5 \%)$ & $2(3.6 \%)$ & 0.63 \\
\hline Adrenal gland & $2(3.6 \%)$ & $3(5.4 \%)$ & 0.66 \\
\hline Others & $2(3.6 \%)$ & $2(3.6 \%)$ & 0.99 \\
\hline \multicolumn{4}{|c|}{ Performance status at beginning of $2 \mathrm{nd}$ line treatment } \\
\hline $0-1$ & $29(52.7 \%)$ & $38(67.9 \%)$ & 0.10 \\
\hline 2 & $21(38.2 \%)$ & $15(26.8 \%)$ & 0.20 \\
\hline$>2$ & $5(9.1 \%)$ & $3(5.3 \%)$ & 0.45 \\
\hline
\end{tabular}

${ }^{*}$ IQR: interquartile range; CNS: central nervous system.

excluded the patients who were nonadenocarcinoma, without EGFR mutation and unknown EGFR status. There were 55 patients who received 1st TKIs/2nd chemotherapy and 56 patients who received the 1 st chemotherapy/2nd TKIs. The general data in two groups was presented in Table 1. There was no difference in two groups including age, gender, smoking status, performance status, and EGFR mutation status.

\subsection{Outcomes Analyses}

\subsubsection{Response}

Response to 1st-Line Treatment. The disease control rate $(81.8 \%$ versus $89.3 \%, p=0.26)$ and response rate $(54.5 \%$ versus $41.7 \%, p=0.16$ ) to 1st-line treatment of two groups were not significantly different. Nevertheless, stable disease rate $(47.6 \%$ versus $27.3 \%, p=0.02$ ) of 1 st-line $\mathrm{TKI} / 2$ nd-line chemotherapy was better than that of another group (Table 2).

3.2.2. Patients Receiving 2nd-Line Treatment. Of 55 patients who received 1st-line TKI/2nd-line chemotherapy, only 27 patients received 2nd-line treatment; meanwhile, among 56 patients who received 1st-line chemotherapy/2nd-line TKIs, most patients $(n=52)$ could receive 2 nd-line treatment. The rate of patients who received 2nd-line treatment was significantly different (49.1\% versus $92.9 \%, p<0.0001)$ between 1st-line TKI/2nd-line chemotherapy group and 1stline chemotherapy/2nd-line TKIs group.

3.2.3. Response to 2 nd-Line Treatment. The response rate (11.1\% versus $25 \%, p=0.15)$ and stable disease rate $(33.3 \%$ versus $46.2 \%, p=0.27$ ) to 2 nd-line treatment of two groups were not significantly different. Nevertheless, disease control rate $(44.4 \%$ versus $71.2 \%, p=0.02)$ to 2 nd-line treatment of 1st-line TKI/2nd-line chemotherapy group was worse than that of another group (Table 2).

3.2.4. Survival. Among all included patients $(n=111)$, the PFS of patients who received 1st-line chemotherapy/2ndline TKIs was similar to that of another group (median PSF: 214 versus 188 days, hazard ratio (HR): 0.69; 95\% confidence interval (CI): $0.46-1.01 ; p=0.06$, Figure 1(a)). The OS was significantly different between two groups (1stline chemotherapy/2nd-line TKIs versus 1st-line TKIs/2ndline chemotherapy: 662 versus 390 days, HR: 0.34; 95\% CI: $0.21-0.55 ; p<0.0001$, Figure 1(b)). 
TABLE 2: Outcomes of stage IV, mutant EGFR lung adenocarcinoma patients who received 2nd-line versus 1st-line chemotherapy.

\begin{tabular}{|c|c|c|c|}
\hline Outcome & $\begin{array}{l}\text { 1st TKIs/2nd chemotherapy } \\
\qquad N=55\end{array}$ & $\begin{array}{l}\text { 1st chemotherapy/2nd TKIs } \\
\qquad N=56\end{array}$ & $p$ value \\
\hline \multicolumn{4}{|l|}{ Response to 1st-line treatment, $n(\%)$} \\
\hline Disease control $(\mathrm{PR}+\mathrm{SD})$ & $45(81.8 \%)$ & $50(89.3 \%)$ & 0.26 \\
\hline PR & $30(54.5 \%)$ & $23(41.7 \%)$ & 0.16 \\
\hline $\mathrm{SD}$ & $15(27.3 \%)$ & $27(47.6 \%)$ & 0.02 \\
\hline $\mathrm{PD}$ & $10(18.2 \%)$ & $6(10.7 \%)$ & 0.26 \\
\hline \multirow[t]{2}{*}{ Patients received 2nd-line treatment } & $27(49.1 \%)$ & $52(92.9 \%)$ & $<0.0001$ \\
\hline & $N=27$ & $N=52$ & \\
\hline \multicolumn{4}{|l|}{ Response to 2nd-line treatment, $n(\%)$} \\
\hline Disease control $(\mathrm{PR}+\mathrm{SD})$ & $12(44.4 \%)$ & $37(71.2 \%)$ & 0.02 \\
\hline $\mathrm{PR}$ & $3(11.1 \%)$ & $13(25 \%)$ & 0.15 \\
\hline $\mathrm{SD}$ & $9(33.3 \%)$ & $24(46.2 \%)$ & 0.27 \\
\hline $\mathrm{PD}$ & $15(55.6 \%)$ & $15(28.8 \%)$ & 0.02 \\
\hline \multicolumn{4}{|l|}{ Survival time, days, median (IQR)* } \\
\hline OS & $390(254-499)$ & $662(342-773)$ & $<0.0001$ \\
\hline PFS to 1st-line treatment & $188(102-276)$ & $214(142-310)$ & 0.06 \\
\hline PFS to 2nd-line treatment & $85(59-148)$ & $199(67-354)$ & $<0.0001$ \\
\hline
\end{tabular}

* IQR: interquartile range; PR; partial response; SD: stable disease; PD: progressive disease; OS: overall survival time; PFS: progression-free survival.

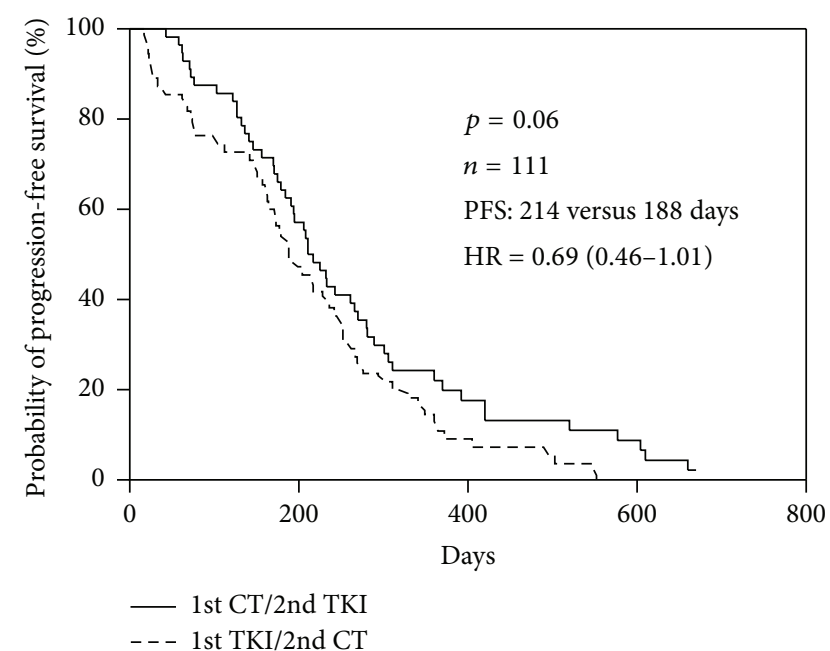

(a)

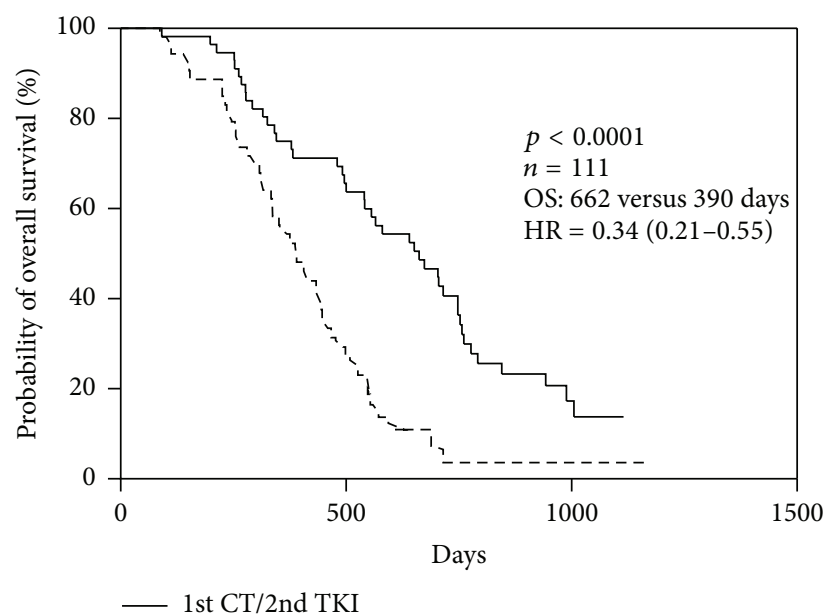

(b)

FIGURE 1: Kaplan-Meier curve of (a) progression-free survival (PFS) and (b) overall survival (OS) of 1st-line treatment for all patients $(n=111$ ) receiving 1st-line chemotherapy/2nd-line TKIs and 1st-line TKIs/2nd-line chemotherapy.

Among patients with $L 858 R$ mutation $(n=63)$, both PFS and OS of patients were significantly different between two groups (Figure 2(a), median PSF: 252 versus 151 days, hazard ratio (HR): $0.11 ; 95 \%$ confidence interval (CI): $0.06-0.21 ; p=$ 0.0001; Figure 2(b), 1st-line chemotherapy/2nd-line TKIs versus 1st-line TKIs/2nd-line chemotherapy: 704 versus 308 days, HR: 0.28; 95\% CI: 0.15-0.53; $p=0.0001$, Figure $1(\mathrm{~b})$ ).

\subsection{Univariate Analysis of Clinical Factors for PFS and OS}

3.3.1. Among all Patients. The median PFS and OS were 198 and 497 days, respectively. Among the clinical factors examined, poor PS, positive smoking history, and 2nd-line chemotherapy treatment and EGFR mutation (L858R versus $19 \mathrm{del}$ ) were significantly related to poor PFS and poor OS (Table 2). 2nd-line chemotherapy was associated with poor PFS (HR: 2.1; 95\% CI: $1.6-4.9 ; p=0.01$ ) and poor OS (HR: 2.3 ; 95\% CI: $1.4-4.7 ; p=0.01$ ) when analyzed with a log-rank test. The Kaplan-Meier survival curves for PFS and OS, according to 1st-line or 2nd-line chemotherapy treatment (Figures 1 and 2), revealed a clear trend toward poor survival outcomes for those patients with 2nd-line chemotherapy (Table 3).

3.3.2. Among Patients with Known EGFR (L858R) Mutation. To take EGFR mutation into consideration, the results were 


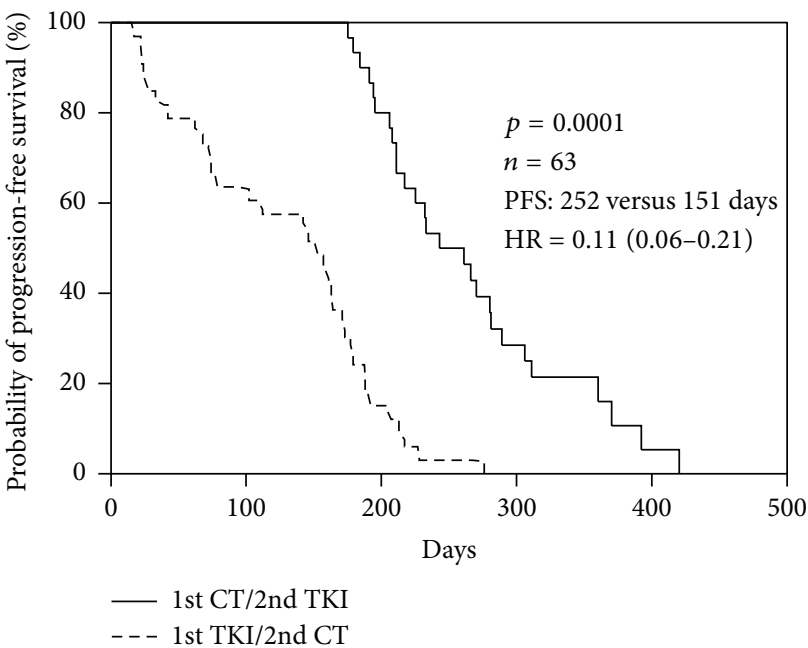

(a)

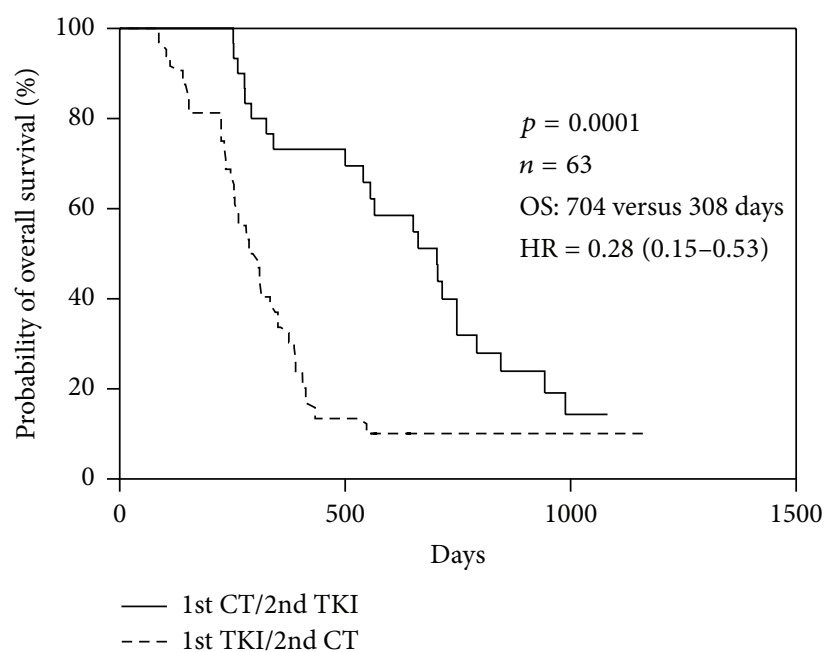

(b)

FIGURE 2: Kaplan-Meier curve of (a) progression-free survival (PFS) and (b) overall survival (OS) of 1st-line treatment for L858R mutation patients $(n=63)$ receiving 1st-line chemotherapy/2nd-line TKIs and 1st-line TKIs/2nd-line chemotherapy.

TABLE 3: Clinical molecular factors for progression-free and overall survival in all patients $(n=111)$ and patients with known $L 858 R$ mutation $(n=63)$ by univariate analysis.

\begin{tabular}{lcccccccc}
\hline & \multicolumn{3}{c}{ All cases $(n=111)$} & \multicolumn{4}{c}{ Patients with known L858R mutation $(n=63)$} \\
& \multicolumn{2}{c}{ Progression-free survival } & \multicolumn{2}{c}{ Overall survival } & \multicolumn{2}{c}{ Progression-free survival } & Overall survival \\
& HR $(95 \% \mathrm{CI})$ & $p$ value & HR $(95 \% \mathrm{CI})$ & $p$ value & HR $(95 \% \mathrm{CI})$ & $p$ value & HR $(95 \%$ CI) & $p$ value \\
\hline Age (y) & $1.2(0.7-1.8)$ & 0.56 & $1.4(0.8-2.2)$ & 0.32 & $1.2(0.6-2.5)$ & 0.7 & $1.1(0.5-2.2)$ & 0.8 \\
Gender & $1.4(0.9-2.2)$ & 0.14 & $1.3(0.8-2.3)$ & 0.22 & $1.5(0.8-2.7)$ & 0.4 & $1.6(0.8-2.9)$ & 0.4 \\
PS >2 & $2.9(1.3-6.1)$ & 0.02 & $6.8(2.9-11.5)$ & $<0.001$ & $5.4(1.6-12.5)$ & 0.01 & $12.1(6.7-15)$ & $<0.001$ \\
Smoking & $2.3(1.3-3.7)$ & 0.03 & $2.3(1.2-3.8)$ & 0.01 & $3.7(1.5-7.5)$ & 0.01 & $3.3(1.1-7.2)$ & 0.001 \\
2nd-line C/T & $2.1(1.6-4.9)$ & 0.01 & $2.3(1.4-4.7)$ & 0.01 & $3.9(1.7-6.8)$ & 0.001 & $4.3(2.0-8.7)$ & 0.001 \\
EGFR mutation $(L 858 R)$ & $2.4(1.3-5.6)$ & 0.02 & $2.1(1.4-4.9)$ & 0.01 & - & - & - & - \\
\hline
\end{tabular}

EGFR: epidermal growth factor receptor gene; HR: hazard ratio; CI: confidence interval; PS: performance status; C/T: chemotherapy; $p$ values were obtained by log-rank test.

also analyzed in the subset of patients with known $E G F R$ $(L 858 R)$ mutation $(n=63)$. Among the clinical factors examined, poor PS, positive smoking history, and 2nd-line chemotherapy significantly correlated with poor PFS and poor OS (Table 3).

3.4. Multivariate Analysis of Factors for PFS and OS. To determine independent factors for PFS and OS, multivariate analysis was performed and included significant factors determined in univariate analysis (Table 4). 2nd-line chemotherapy was significant for PFS (OR: 1.7; 95\% CI: 1.573.89; $p=0.03$ ) and OS (OR: 1.8; 95\% CI: 1.4-3.9; $p=0.01$ ). Similarly, EGFR mutation with $L 858 R$ was also significant for PFS (OR: 2.2; 95\% CI: 1.7-4.5; $p=0.02$ ) and OS (OR: 2.6; $95 \%$ CI: $1.2-5.6 ; p=0.01)$. These data indicate that L858R and 2nd-line chemotherapy were significant factors for PFS and OS, independent of other confounding factors.

\section{Discussion}

As IPASS data revealed NSCLC patients with mutant EGFR had a high response rate to 1st-line TKI treatment, 30\% patients with lung adenocarcinoma and EGFR mutation had no response to 1st-line EGFR-TKI treatment [8]. Recently, TORCH study reported that first-line erlotinib followed at progression by cisplatin-gemcitabine was significantly inferior in terms of overall survival compared with the standard sequence of first-line chemotherapy followed by erlotinib in unselected patients with advanced NSCLC [12]. Therefore, the 1st-line treatment to patients with mutant $E G F R$ advanced lung adenocarcinoma is an important issue whether chemotherapy or EGFR-TKIs selected. Our study demonstrates that the 1st-line chemotherapy followed by EGFR-TKIs in patients with EGFR mutation advanced lung adenocarcinoma had a better OS than that of the 1st-line TKIs followed by 2nd-line chemotherapy in these patients 
TABLE 4: Final multivariate model for progression-free survival and overall survival.

\begin{tabular}{|c|c|c|c|c|}
\hline & \multicolumn{2}{|c|}{ Progression-free survival } & \multicolumn{2}{|c|}{ Overall survival } \\
\hline & OR $(95 \% \mathrm{CI})$ & $p$ value & OR $(95 \% \mathrm{CI})$ & $p$ value \\
\hline Poor PS: $>2$ & $1.5(0.5-4.2)$ & 0.58 & $1.9(0.81-3.82)$ & 0.49 \\
\hline Smoking & $1.3(0.6-4.7)$ & 0.49 & $1.4(0.7-2.2)$ & 0.31 \\
\hline 2nd-line $\mathrm{C} / \mathrm{T}$ & $1.7(1.51-3.89)$ & 0.03 & $1.8(1.4-3.91)$ & 0.01 \\
\hline EGFR mutation ( $(2858 R$ to $19 \mathrm{Del})$ & $2.2(1.7-4.5)$ & 0.02 & $2.6(1.2-5.6)$ & 0.01 \\
\hline
\end{tabular}

OR: odds ratio; CI: confidence interval; PS: performance status; C/T: chemotherapy; EGFR: epidermal growth factor receptor gene. $p$ values were obtained by multinominal logistic regression analysis.

(median: 662 versus 390 days, $p<0.0001$; Figure 1(b) and Table 2). Most of these patients (52/56, 92.9\%) could shift to 2nd-line TKIs when disease progressed after 1st-line chemotherapy, meanwhile, less patients $(27 / 55,49.1 \%)$ could receive 2 nd-line chemotherapy when disease progressed after 1st-line TKIs treatment. The PFS to 2nd-line treatment in 1 st chemotherapy/2nd TKIs group is significantly better than that of 1st TKIs/2nd chemotherapy group (median: 199 versus 85 days, $p<0.0001$; Table 2); similar trend was found in PFS to 1st-line treatment despite being without statistical significance (median: 214 versus 188 days, $p=0.06$; Figure 1 (a) and Table 2).

All of patients in this study had mutant EGFR advanced lung adenocarcinoma and both groups had similar performance status. The choice of initial therapy is influenced by financial problem and the toxicity of chemotherapy. There has been no comparative data between first- and secondline EGFR-TKIs in patients with activating EGFR mutations so far. However, tumor response rates to second-line EGFRTKIs are inconsistent. It may be due to the effect of firstline chemotherapy on the abundance of tumor cells with activating EGFR mutations.

Despite IPASS or West Japan Oncology Group trial revealing that the PFS is better in 1st-line TKIs than chemotherapy, the OS is not significantly different. The possible reason may be caused by the further systemic therapy which may affect the final survival outcomes. Therefore, we also conducted a prospectively randomized study to investigate the differential influence of prior chemotherapy on the efficacy of erlotinib in patients with advanced non-small-cell lung cancer (ClinicalTrials.gov Identifier: NCT01204307). The prospective study had been completed and will be reported in near future. Nevertheless, from current study, we supported that PFS could be a guide of treatment response evaluation to further treatment after TKIs or chemotherapy as the 1stline treatment. Some studies also showed prolonged PFS with 2nd-line TKIs after 1st-line chemotherapy [13-15]. The benefit of TKIs in mutant EGFR NSCLC patients is confirmed in 1stline or 2nd-line therapy. It is reasonable to switch another treatment when initial treatment (with TKI or chemotherapy) was failure. TKIs had adverse effect of skin rash, diarrhea, pulmonary toxicity, and hepatic toxicity [16]. Most of adverse effect is mild and tolerable in old age patients [17]. When 1st-line TKIs are prescribed in advanced NSCLC, the disease progression is noted due to secondary mutation of EGFR and $M E T$ oncogene amplification $[18,19]$. The further systemic therapy will be given. There is no randomized clinical trial to compare different regimens of 2nd-line chemotherapy after 1st-line TKIs treatment.

Prior study reported that, after 1st-line platinum-based doublet chemotherapy, the efficiency of 2nd-line treatment between TKIs and chemotherapy after chemotherapy was no different [20]. Another report also presented that the TKIs can be a choice of maintenance therapy after four cycles of chemotherapy [21]. TKIs and chemotherapy both are still effective as 2nd-line therapy. In clinical practice, the crossover treatment is usually noted when disease progresses. In TORCH trial, the standard treatment (1st chemotherapy/2nd TKIs) is better than experimental treatment (1st TKIs/2nd chemotherapy) in unselected NSCLC patients. However, there is small portion of mutant EGFR patients $(N=39)$ in TORCH study. The total PFS is longer in standard treatment but there is not statistically significant (HR: 1.71, 95\% CI: 0.82 to 3.60) in mutant EFGR NSCLC patients [12].

In mutant EGFR advanced lung adenocarcinoma patients, the efficiency of TKIs has been demonstrated in other trials $[8,9,12]$. However, the other systemic therapy contributes the survival outcome and makes the similar OS of patients who received 1st line TKIs or 1st line chemotherapy [8]. The timing of chemotherapy may be important. The 2ndline chemotherapy after TKIs may not be as potent as 1st-line chemotherapy. The major cause is the nature course of disease progression and the toxicity of chemotherapy. It includes malnutrition, decondition, tumor enlargement, metastasis, and performance deterioration. All of the above reasons will affect the interval, dose, and regimen of chemotherapy. They are associated with the response of chemotherapy. Another reason is the consideration of adverse effect and patient's willingness. Many patients could not complete course of 2nd-line chemotherapy after 1st-line TKIs treatment. Only $67.5 \%$ patients continue 2nd-line chemotherapy in TKIs group and the other group is $94.6 \%$ patients receiving 2ndline TKIs after chemotherapy [22]. Our data also revealed similar result; furthermore, we found most patients receiving 1st TKI treatment usually suffering rapid progression and worse performance status when disease progressed. The poor performance status of these patients caused them no chances to receive further chemotherapy! The survival duration will be shorted if there is no further systemic therapy because of the rapid progression and poor performance status. In addition, in those patients who could receive 2 nd-line treatment, better disease control rate and PFS to 2nd-line treatment in 1st chemotherapy/2nd TKIs group were also found in our data (Table 2). These reasons may explain that 
the 1st chemotherapy/2nd TKIs is better than 1st TKIs/2nd chemotherapy group.

In Taiwan, the National Health Insurance provided TKIs use in mutant EGFR NSCLC. However, the duration of detection of mutation status of EGFR and application of TKIs usually costs two weeks at least. The initial therapy may be delayed because of those reasons. The 1st-line chemotherapy can reduce the timing of initial treatment. The further decision of TKIs use can be done after four- to six-cycle chemotherapy according to American Society of Clinical Oncology Clinical Practice Guideline [23].

In addition, possible and important reasons to explain our data are clinical trials of Lux-Lung 3 and 6 by Yang et al. [24]. The results of the trials revealed that the outcomes of patients who harbored 19Del and L858R were different from 1stline EGFR-TKI treatment. In subgroup analysis of the trials, patients with L858R may not benefit from 1st-line EGFR-TKI; reversely, these subgroup patients may had better outcome from 1st-line chemotherapy! Similarly, in our current study, most patients harbored EGFR mutation with $L 858 R$ in both groups (up to 55-58\%); meanwhile, less patients were with 19Del (38-41\%). This distribution of different EGFR mutation may explain why the outcomes of our patients were poor in those patients who received chemotherapy as the second-line treatment.

Limitation. First, it was not a prospective study; the character of the current study may cause bias. Second, the numbers of the current study were small so the bias should be considered. As presentation of Table 1, we listed the progression sites from each 1st-line treatment and PS at the beginning of 2nd-line treatment. Patients in 1st-line TKI group had more central nervous system (CNS) metastasis and poor performance in trend but statistical insignificance was found. The relatively small numbers in this study may explain the condition. However, patients with 1st-line TKI revealed the trend of worse performance at the beginning of 2nd-line treatment from the study. Similarly, small numbers may cause statistical insignificance. Despite the baseline condition and underlying disease of patients being balanced in Table 1, this study was not a random control trial. Some potential or unknown factors may be unbalanced due to the nonrandomized setting. Therefore, the occurrence of a skewed population cannot be excluded. The results of this study need to be confirmed by large-scale prospective randomized studies.

\section{Conclusion}

According to our study, the better overall survival is found in 1st chemotherapy/2nd TKIs group, especially among those patients with $L 858 R$ mutation. The effect of chemotherapy may be better as an initial therapy of NSCLC, especially in mutant EGFR with $L 858 R$ group. It is a consideration for the choice of systemic therapy sequence. In addition, similar to Lux-Lung 3 and 6 trials, our data also provided a different but important consideration of 1st-line chemotherapy treatment in patients with L858R EGFR mutation. The further perspective study shall be evaluated in the future. Although it is not appropriate to make such a conclusion with this study, a prospective randomized study is warranted to confirm these findings. Finally, the possible larger response rate seen in this Asian population and in others reported in the literature is worthy of further study both in terms of biomarkers and possible differences in pharmacogenomics.

\section{Disclosure}

The funders had no role in study design, data collection and analysis, decision to publish, or preparation of the paper. No additional external funding was received for this study.

\section{Conflict of Interests}

All authors do not have any conflict of interests.

\section{Authors' Contribution}

Fu-Tsai Chung, Ming-Yun Ho, and Han-Pin Kuo conceived, designed, and performed the study, analyzed the data, and wrote the paper. Tsai-Yu Wang, Chih-Hsi Kuo, Hao-Cheng Chen, Chun-Hwa Wang, Yueh-Fu Fang, Meng-Heng Hshieh, Shu-Min Lin, Chih-Teng Yu, and Horng-Chyuan Lin helped to enroll the patients and analyzed the data. Fu-Tsai Chung and Ming-Yun Ho contributed equally to the work on this project as first authors.

\section{Acknowledgments}

The authors thank Clinical Informatics and Medical Statistics Research Center of Chung Gung University and Hospital for providing the recommendations of statistical analyses. This study was supported in part by grants from Chang Gung Memorial Hospital (CMRPG3B0981, CMRPG3B0982, and CMRPG3B0983 by Drs. Fu-Tsai Chung and Han-Pin Kuo).

\section{References}

[1] S. Sun, J. H. Schiller, and A. F. Gazdar, "Lung cancer in never smokers-a different disease," Nature Reviews Cancer, vol. 7, no. 10, pp. 778-790, 2007.

[2] R. S. Herbst, J. V. Heymach, and S. M. Lippman, "Lung cancer," The New England Journal of Medicine, vol. 359, no. 13, pp. 1367$1380,2008$.

[3] L. V. Sequist, D. W. Bell, T. J. Lynch, and D. A. Haber, "Molecular predictors of response to epidermal growth factor receptor antagonists in non-small-cell lung cancer," Journal of Clinical Oncology, vol. 25, no. 5, pp. 587-595, 2007.

[4] F. Ciardiello and G. Tortora, "Drug therapy: EGFR antagonists in cancer treatment," The New England Journal of Medicine, vol. 358, no. 11, pp. 1160-1174, 2008.

[5] T. J. Lynch, D. W. Bell, R. Sordella et al., "Activating mutations in the epidermal growth factor receptor underlying responsiveness of non-small-cell lung cancer to gefitinib," The New England Journal of Medicine, vol. 350, no. 21, pp. 2129-2139, 2004.

[6] R. Rosell, T. Moran, C. Queralt et al., "Screening for epidermal growth factor receptor mutations in lung cancer," The New England Journal of Medicine, vol. 361, no. 10, pp. 958-967, 2009.

[7] W. Pao, V. Miller, M. Zakowski et al., "EGF receptor gene mutations are common in lung cancers from 'never smokers' and are 
associated with sensitivity of tumors to gefitinib and erlotinib," Proceedings of the National Academy of Sciences of the United States of America, vol. 101, no. 36, pp. 13306-13311, 2004.

[8] T. S. Mok, Y.-L. Wu, S. Thongprasert et al., "Gefitinib or carboplatin-paclitaxel in pulmonary adenocarcinoma," The New England Journal of Medicine, vol. 361, no. 10, pp. 947-957, 2009.

[9] T. Mitsudomi, S. Morita, Y. Yatabe et al., "Gefitinib versus cisplatin plus docetaxel in patients with non-small-cell lung cancer harbouring mutations of the epidermal growth factor receptor (WJTOG3405): an open label, randomised phase 3 trial," The Lancet Oncology, vol. 11, no. 2, pp. 121-128, 2010.

[10] E. A. Eisenhauer, P. Therasse, J. Bogaerts et al., "New response evaluation criteria in solid tumours: revised RECIST guideline (version 1.1)," European Journal of Cancer, vol. 45, no. 2, pp. 228247, 2009.

[11] F.-T. Chung, K.-Y. Lee, C.-W. Wang et al., “Tumor-associated macrophages correlate with response to epidermal growth factor receptor-tyrosine kinase inhibitors in advanced nonsmall cell lung cancer," International Journal of Cancer, vol. 131, no. 3, pp. E227-E235, 2012.

[12] C. Gridelli, F. Ciardiello, C. Gallo et al., "First-line erlotinib followed by second-line cisplatin-gemcitabine chemotherapy in advanced non-small-cell lung cancer: the TORCH randomized trial," Journal of Clinical Oncology, vol. 30, no. 24, pp. 3002-3011, 2012.

[13] L. Zhang, S. Ma, X. Song et al., "Gefitinib versus placebo as maintenance therapy in patients with locally advanced or metastatic non-small-cell lung cancer (INFORM; C-TONG 0804): a multicentre, double-blind randomised phase 3 trial," The Lancet Oncology, vol. 13, no. 5, pp. 466-475, 2012.

[14] K. Takeda, T. Hida, T. Sato et al., "Randomized phase III trial of platinum-doublet chemotherapy followed by gefitinib compared with continued platinum-doublet chemotherapy in Japanese patients with advanced non-small-cell lung cancer: results of a West Japan Thoracic Oncology Group Trial (WJTOG0203)," Journal of Clinical Oncology, vol. 28, no. 5, pp. 753-760, 2010.

[15] W. Brugger, N. Triller, M. Blasinska-Morawiec et al., "Prospective molecular marker analyses of EGFR and KRAS from a randomized, placebo-controlled study of erlotinib maintenance therapy in advanced non-small-cell lung cancer," Journal of Clinical Oncology, vol. 29, no. 31, pp. 4113-4120, 2011.

[16] R. J. Cersosimo, "Gefitinib: an adverse effects profile," Expert Opinion on Drug Safety, vol. 5, no. 3, pp. 469-479, 2006.

[17] M. Maemondo, Y. Minegishi, A. Inoue et al., "First-line gefitinib in patients aged 75 or older with advanced non-small cell lung cancer harboring epidermal growth factor receptor mutations. NEJ 003 study," Journal of Thoracic Oncology, vol. 7, no. 9, pp. 1417-1422, 2012.

[18] S. Kobayashi, T. J. Boggon, T. Dayaram et al., "EGFR mutation and resistance of non-small-cell lung cancer to gefitinib," The New England Journal of Medicine, vol. 352, no. 8, pp. 786-792, 2005.

[19] J. A. Engelman, K. Zejnullahu, T. Mitsudomi et al., "MET amplification leads to gefitinib resistance in lung cancer by activating ERBB3 signaling," Science, vol. 316, no. 5827, pp. 1039-1043, 2007.

[20] T. Ciuleanu, L. Stelmakh, S. Cicenas et al., "Efficacy and safety of erlotinib versus chemotherapy in second-line treatment of patients with advanced, non-small-cell lung cancer with poor prognosis (TITAN): a randomised multicentre, open-label, phase 3 study," The Lancet Oncology, vol. 13, no. 3, pp. 300-308, 2012.

[21] F. Cappuzzo, T. Ciuleanu, L. Stelmakh et al., "Erlotinib as maintenance treatment in advanced non-small-cell lung cancer: a multicentre, randomised, placebo-controlled phase 3 study," The Lancet Oncology, vol. 11, no. 6, pp. 521-529, 2010.

[22] M. Maemondo, A. Inoue, K. Kobayashi et al., "Gefitinib or chemotherapy for non-small-cell lung cancer with mutated EGFR," The New England Journal of Medicine, vol. 362, no. 25, pp. 2380-2388, 2010.

[23] C. G. Azzoli, S. Temin, and G. Giaccone, "2011 focused update of 2009 American society of clinical oncology clinical practice guideline update on chemotherapy for stage IV non-small-cell lung cancer," Journal of Oncology Practice, vol. 8, no. 1, pp. 6366, 2012.

[24] J. C.-H. Yang, Y.-L. Wu, M. Schuler et al., "Afatinib versus cisplatin-based Chemotherapy for EGFR mutation-positive lung adenocarcinoma (LUX-Lung 3 and LUX-Lung 6): analysis of overall survival data from two randomised, phase 3 trials," The Lancet Oncology, vol. 16, no. 2, pp. 141-151, 2015. 


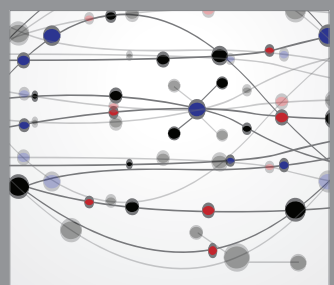

The Scientific World Journal
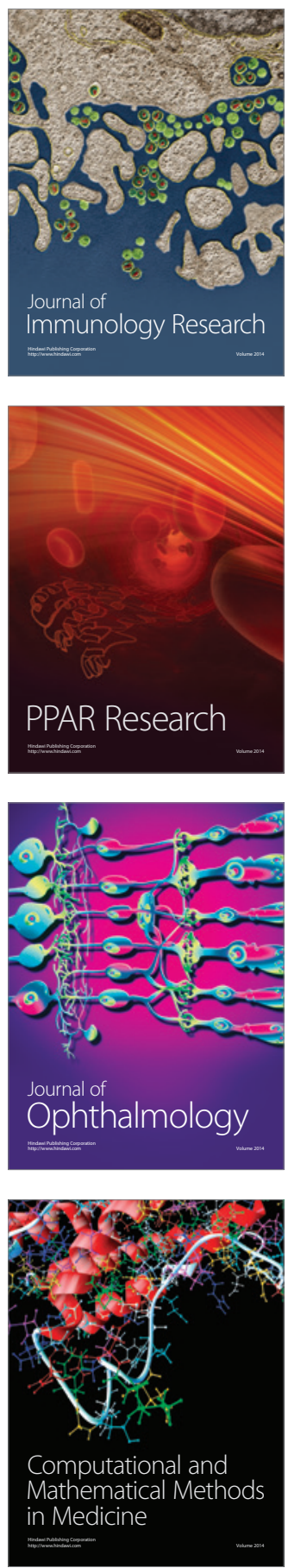

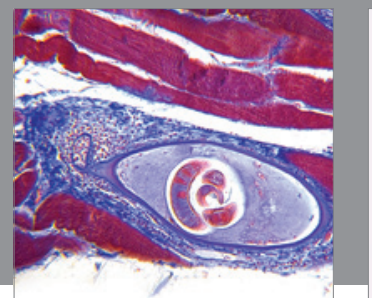

Gastroenterology

Research and Practice
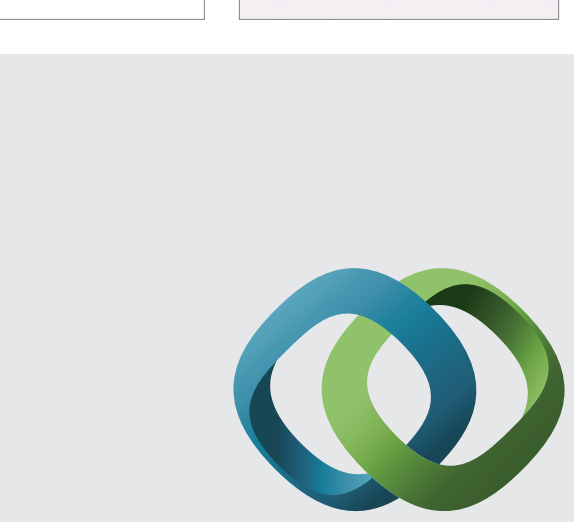

\section{Hindawi}

Submit your manuscripts at

http://www.hindawi.com
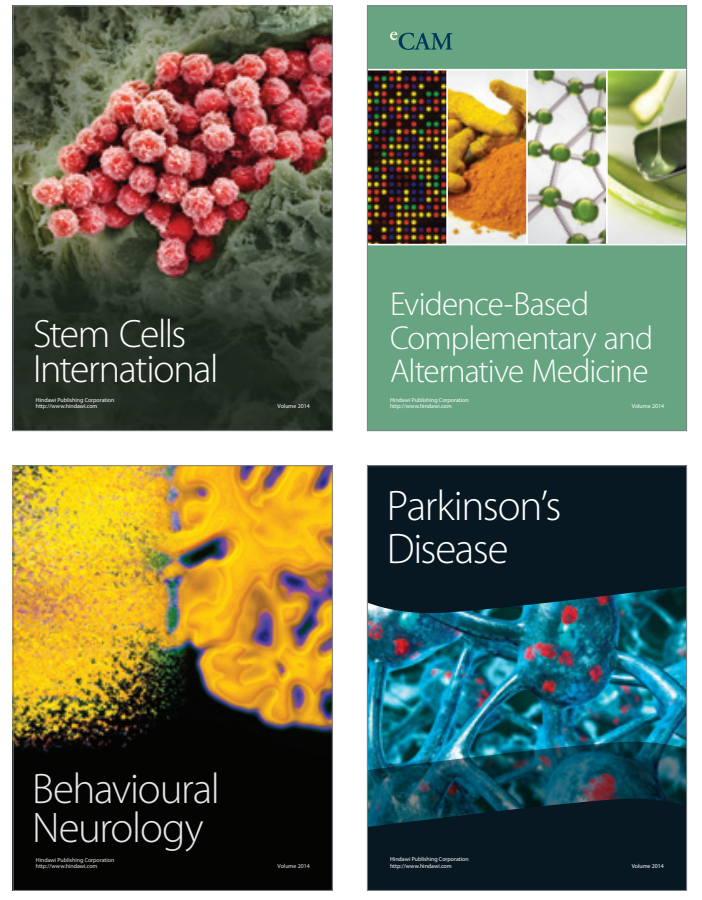
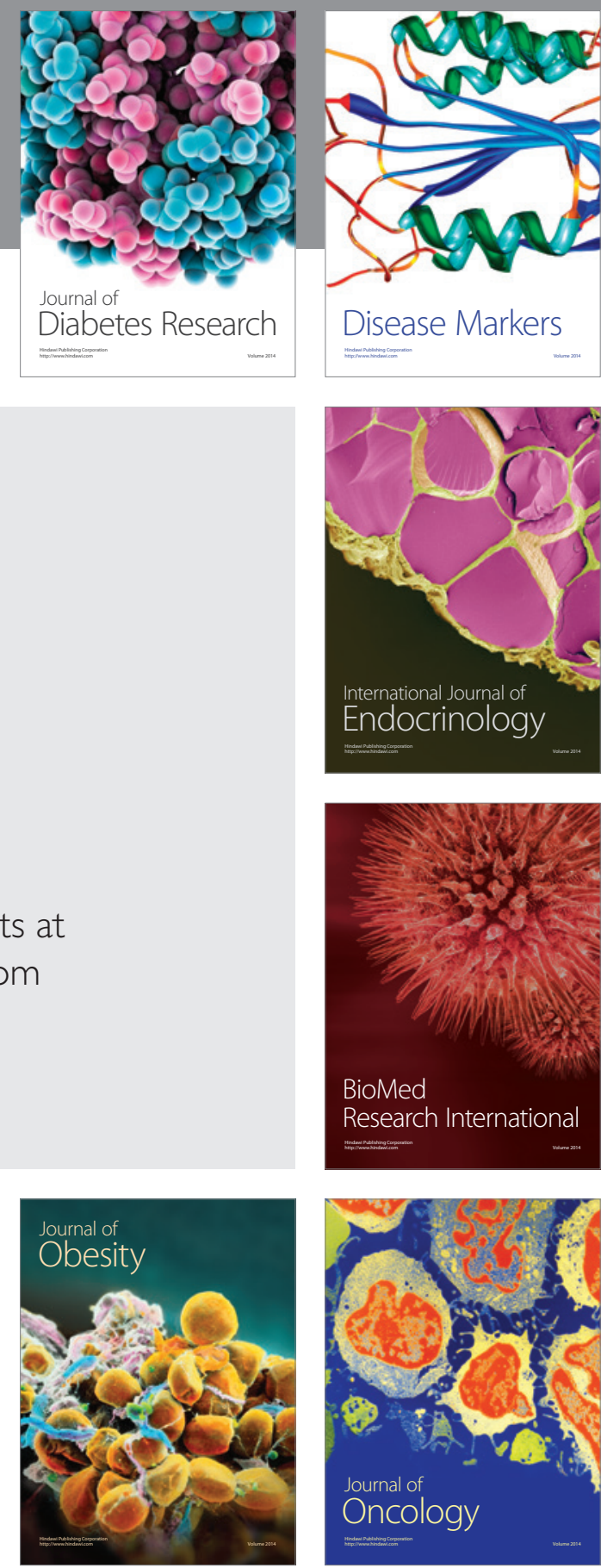

Disease Markers
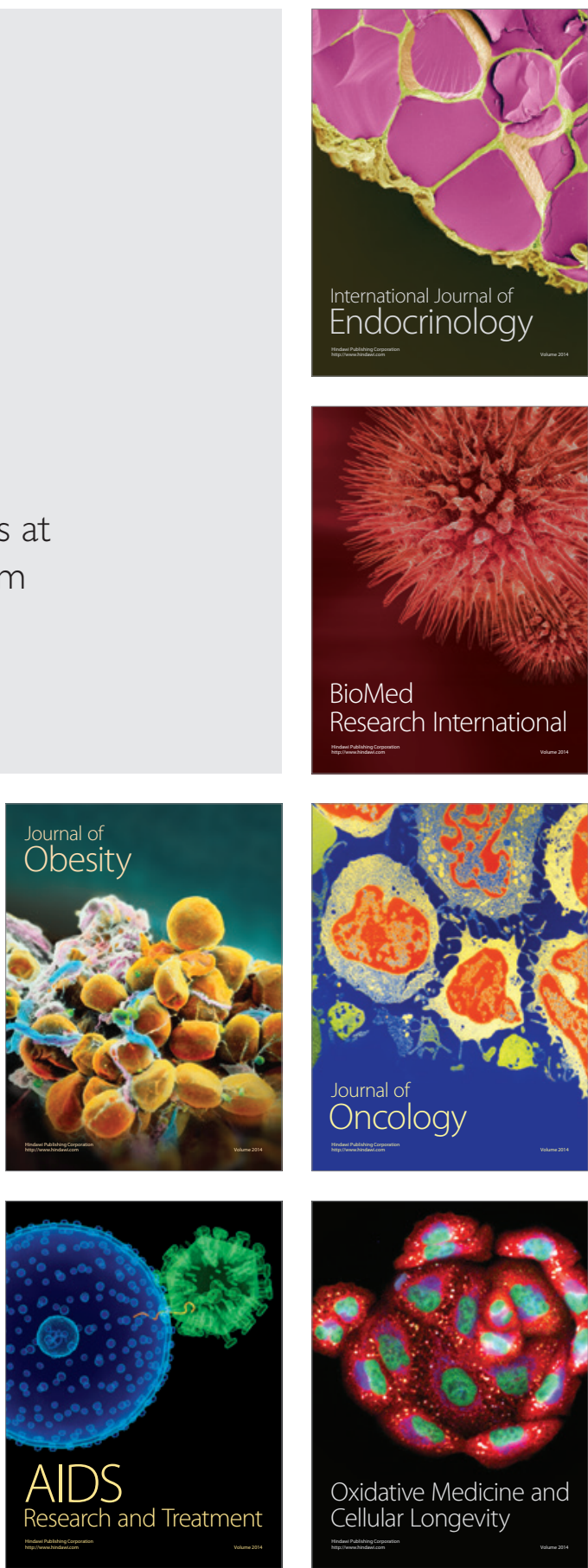